\title{
Effect of ions on conductivity and permittivity in the Polar Mesosphere Summer Echoes region
}

\author{
Safi Ullah ${ }^{1,2}$, HaiLong Li ${ }^{1,2 *}$, Abdur Rauf1,2, Lin Meng ${ }^{1,2}$, Bin Wang ${ }^{1,2}$, ShuCan Ge ${ }^{1,3}$, and MaoYan Wang ${ }^{4}$ \\ 'School of Electronic Science and Engineering, University of Electronic Science and Technology of China, Chengdu 610054, China; \\ ${ }^{2}$ Terahertz Science and Technology Key Laboratory of Sichuan Province, Chengdu 610054, China; \\ ${ }^{3}$ National Key Laboratory of Electromagnetic Environment, China Research Institute of Radiowave Propagation, Qingdao 266107, China; \\ ${ }^{4}$ School of Physics, University of Electronic Science and Technology of China, Chengdu 610054, China
}

\section{Key Points:}

- At PMSE (polar mesosphere summer echoes) altitudes, the bite-out condition contains high dust and low electron density.

- In the absence of the bite-out condition, the ion effects on conductivity and permittivity are not significant.

- In the presence of the bite-out condition, the ion effects on conductivity and permittivity are significant and cannot be excluded.

Citation: Ullah, S., Li, H. L., Rauf, A., Meng, L., Wang, B., Ge, S. C. and Wang, M. Y. (2021). Effect of ions on conductivity and permittivity in the Polar Mesosphere Summer Echoes region. Earth Planet. Phys., 5(2), 196-204. http://doi.org/10.26464/epp2021016

\begin{abstract}
For the first time, the effect of ions on complex conductivity and permittivity of dusty plasma at Polar Mesosphere Summer Echoes (PMSE) altitude is analyzed. Because of ions higher mass and smaller thermal velocity, generally, their effects are not considered in the study of electromagnetic properties of dusty plasmas. In this study, we modified the equations of conductivity and permittivity by adding the effect of ions. In the PMSE altitude region between 80 and $90 \mathrm{~km}$, a local reduction in electron density (i.e., an electron biteout), is produced by electron absorption onto dust particles. The bite-out condition contains high dust density and smaller electron density. From simulation results in comparatively strong bite-out conditions, we found that the ion effects on conductivity become significant with smaller dust size, lower electron temperature, and lower neutral density. For comparatively weak bite-out conditions, the ion effects on conductivity become significant with larger dust size, higher electron temperature, and higher neutral density. On the other hand, for different dust sizes, electron temperatures and neutral density, the ion effects on complex permittivity become significant only in very strong bite-out conditions. Based on these simulation results, we conclude that, in the absence of electron bite-out conditions, the effect of ions on complex conductivity and permittivity is not significant and can be ignored. However, during bite-out conditions, the effect of ions becomes significant and cannot be ignored because it significantly changes the conductivity and permittivity of dusty plasmas.
\end{abstract}

Keywords: PMSE; electromagnetic waves; collision and charging effect; conductivity and permittivity

\section{Introduction}

The mesosphere at polar latitudes is a highly dynamic and complex region where chemical and physical processes take place, where the disintegration of meteors between 50 and $90 \mathrm{~km}$ altitude forms natural dusty plasmas. Human activity and climate change cause an increase of methane concentration, leading to elevated water vapor content and lowered mesospheric temperatures (Rapp and Lübken, 2004). During summer, the temperature of the polar mesopause region falls to about $130 \mathrm{~K}$ (Von Zahn and Meyer, 1989; Lübken, 1999). Due to this low temperature, ice particles form and grow to produce noctilucent clouds (NLC).

NLC provide direct evidence of the formation of ice-coated met-

Correspondence to: H. L. Li, hailong703@163.com

Received 18 OCT 2020; Accepted 22 DEC 2020.

Accepted article online 27 JAN 2021.

(C)2021 by Earth and Planetary Physics. eoric dust particles from the freezing of water vapor, with radii exceeding $20 \mathrm{~nm}$. At polar latitudes, nanometer-size dust particles produce remarkably strong radar echoes due to electron density irregularities, called polar mesosphere summer echoes (PMSE) (Lübken, 1999), and NLC can even be observed optically below the PMSE layer (Von Zahn and Bremer, 1999). In the D-region of the ionosphere, one possible reason for electron density irregularity and hence for PMSE might be the extra ionization produced by highly energetic particle precipitation (Rauf et al., 2018). Recently, Ge et al. (2020) presented the effect of energetic particle precipitation on modulated PMSE. The dust particles are supposed to be involved in the creation of PMSE, although the layer is not visible, it can be observed by strong radio reflections with radars operating between $50 \mathrm{MHz}$ and $1.3 \mathrm{GHz}$. Interest in the study of dusty plasmas in the mesosphere has significantly expanded due to their possible connection with the Earth global warming process. PMSE have been intensively studied for more than 30 years; a detailed review of theory and observations of PMSE can be found in 


\section{Rapp and Lübken (2004).}

In-situ measurements have contributed significantly to the understanding of the polar mesosphere region chemical and physical processes, using multi-range radar with time-space spectral measurements. Moreover, artificial electron heating experiments have been used as an alternative approach to investigate the dusty plasma parameters in the mesosphere region. Using the EISCAT (European Incoherent SCATter) heating facility, Chilson et al. (2000) discovered that PMSE strength was modulated by heating the mesosphere with powerful high-frequency (HF) radio waves (Chilson et al., 2000), further studied by Rapp and Lübken (2000) and later explained by Havnes et al. (2003) and Havnes (2004). Theoretical models successfully explained the PMSE heating effect for HF radar (VHF $224 \mathrm{MHz}$ and UHF $930 \mathrm{MHz}$ ) (Næsheim et al., 2008; Biebricher et al., 2012).

The study of heating effects at low frequencies includes Scales (2004), Chen C and Scales (2005), Biebricher and Havnes (2012) and Senior et al. (2014). In the study of PMSE, Havnes (2004) extended the use of radar by introducing a theory about the role of dust particles in PMSE formation. The dust particles become electrically charged through the attachment of free ionospheric plasma particles and photo-emission of electrons, where the dust size, solar radiation, and plasma parameters determine the average dust charge. Depending on the balance of electron and ion currents at the dust surface, a pure dust particle acquires a small negative charge and a high photoelectric work function. At PMSE altitudes, this effect produces a local reduction in electron density in the surrounding plasma called an electron bite-out (Kassa et al., 2005). However, significant photoionization due to metallic species on the dust surface results in an increase of free electron density, and the dust particle may become positively charged (Weingartner and Draine, 2001). Rocket observations confirmed both positive and negative dust charges (Havnes et al. 1996; Blix, 1999).

It is now commonly accepted that the creation of PMSE is due to the combination of neutral atmospheric turbulence and charged dust particles, however, at polar latitudes the transition of electromagnetic (EM) waves in dusty plasmas is still an open question. In summer conditions at polar latitude, dust particles play a crucial role in the balancing of charge in the mesopause region. Within the PMSE layer, comparatively large concentrations of dust particles produce the electron bite-out condition. Given high enough dust density, the local electron density is significantly reduced due to electron absorption by dust particles (Ulwick et al., 1988). The existence of dust particles is strongly supported by the local reduction in electron density (bite-out). So far, in the vicinity of PMSE, electron bite-outs have been observed routinely with rocket-borne sensors as well as occasionally with ground-based radars (Friedrich et al., 2009; Singer et al., 2011). The corresponding rocket measurements during the STATE (Structure and Atmospheric Turbulence Environment) campaign in 1983 have shown that the electron number density may be depleted by as much as a factor of 10 in the presence of PMSE (Ulwick et al., 1988).

It is revealed that the presence of dust particles are necessary for the creation of PMSE (Chen and Scales, 2005). Thus, to study the mechanics of PMSE, it is of great importance to start with dusty plasmas, which are relevant for a number of applications in laboratory plasmas and modern plasmas technologies, as well as in space plasmas and plasma of the Earth's environment (Havnes et al., 1992). Due to the presence of charged dust particles, the dynamical behaviors of dusty plasma are different from that of ionelectron plasma. Scattering of electromagnetic waves in plasma is a powerful diagnostic method which has been successfully used in the laboratory and active geophysical experiments (Blix, 1999).

Electrical permittivity and conductivity can be used to describe the electromagnetic properties of plasma, with properties of dusty plasmas being different from that of ion-electron plasma. Shi YX et al. (2007) calculated the conductivity and permittivity of weakly-ionized dusty plasmas, while Duan JZ et al. (2012) reported the effect of dust size distribution on complex conductivity of dusty plasmas. Due to higher mass and smaller thermal velocity, the effect of ions was not considered in the previous study of dusty plasma electromagnetic properties, since the presence of ions in the equations did not significantly affect the conductivity and permittivity. The PMSE region $(80-90 \mathrm{~km})$ acts as a natural dusty plasma laboratory where the dust density sometimes increases, and the electron density decreases to form the electron bite-out condition. In the case of high dust density, the electron density is much smaller than the ion density. Until now, the ion effects on electromagnetic properties of dusty plasmas in bite-out conditions has not been studied. For this analysis, we chose a biteout condition at PMSE altitudes because we expect that the charging response factor of ions, plasma-ion frequency, effective collision frequency of ions, and thermal velocity of ions may change significantly. Therefore, in this study, we consider the effect of ions on complex conductivity and permittivity in the PMSE altitude region between 80 and $90 \mathrm{~km}$ in the presence and absence of electron bite-out conditions for the first time. This may yield additional information about the role of ions in the equations of conductivity and permittivity of dusty plasmas.

\section{Effect of Collisions}

Considering weakly-ionized dusty plasma consisting of electrons, ions, neutral atoms and charged dust particles, the kinetic equation for ions can be written as (Ginzburg, 1970):

$$
\frac{\partial f}{\partial t}+u \cdot \nabla_{\mathrm{r}} f+\frac{e}{m_{\mathrm{i}}}(E) \cdot \nabla_{u} f+S=0
$$

where $f, e, m_{i}$ and $v$ are the ion distribution function, charge, mass and speed, respectively; $E$ is the electric field, and $S$ is the Bhatnagar Gross and Krook (BGK) collision term describing the function of the variation of ion distribution. We suppose that from the combination of weak electric field and collision terms, a deviation of $f_{1}$ occurs, as a result, the ions' distribution function $f$ can be written as:

$$
f(u)=f_{0}(u)+\frac{u \cdot f_{1}(u)}{u},
$$

where $f_{0}$ is the Maxwellian distribution function of ions, which is equal to $f_{0}=N_{\mathrm{i}}\left(m_{\mathrm{i}} / 2 \pi k T_{\mathrm{i}}\right)^{3 / 2} \cdot \mathrm{e}^{-m_{\mathrm{i}} v^{2} / 2 k T_{\mathrm{i}}}$ here; $N_{\mathrm{i}}$ and $T_{\mathrm{i}}$ are the ion density and temperature, respectively; $k$ is the Boltzmann constant $\left(1.38 \times 10^{-23} \mathrm{~J} \cdot \mathrm{K}^{-1}\right)$. The perturbation $f_{1}(u)$ is the vector distribution function of ion speed with a direction parallel to $E$ and 
magnitude $\left|f_{1}\right| \ll f_{0}$. Substituting Equation (2) into (1) and neglecting the second-order terms, we obtain:

$$
\frac{\partial f_{1}}{\partial t}+\frac{e E}{m_{\mathrm{i}}} \frac{\partial f_{0}}{\partial u}+v(u) f_{1}=0
$$

The solution of Equation (3) can be written as

$$
f_{1}(u)=\frac{e E N_{\mathrm{i}}}{2 m_{\mathrm{i}} \pi^{3 / 2}[\mathrm{i} \omega+v(u)]}\left(\frac{m_{\mathrm{i}}}{k T_{\mathrm{i}}}\right)^{2} u \mathrm{e}^{-u^{2}} .
$$

In the above equation, $\omega$ is the angular frequency of the EM wave and $u=\sqrt{m_{\mathrm{i}} / 2 k T}$. The relation of current density $\left(j^{\prime}\right)$ with distribution function can be written as:

$$
j^{\prime}=\frac{8 \mathrm{e}^{2} N_{\mathrm{i}} E}{3 \sqrt{\pi} m_{\mathrm{i}}}\left\{\int_{0}^{\infty} \frac{v(u) u^{4} \mathrm{e}^{-u^{2}} \mathrm{~d} u}{\omega^{2}+v^{2}(u)}-\mathrm{i} \omega \int_{0}^{\infty} \frac{u^{4} \mathrm{e}^{-u^{2}} \mathrm{~d} u}{\omega^{2}+v^{2}(u)}\right\} .
$$

The current density and electric field are also related by Ohm's law (Ginzburg, 1970):

$$
j^{\prime}=\sigma_{\text {complex }} E=\left(\sigma_{\mathrm{i}}+\mathrm{i} \omega \varepsilon_{0} X\right) E .
$$

All terms in Equation (5) are already defined in the text. In Equation (6) $\sigma_{i}$ and $x$ are the ion conductivity and electric susceptibility, respectively. Comparing Equations (5) and (6), we can obtain the ion conductivity and permittivity as:

$$
\begin{gathered}
\sigma_{\mathrm{i}}=\varepsilon_{0} \frac{\omega_{\mathrm{pi}}^{2} v_{\mathrm{eff}}}{\omega^{2}+v_{\mathrm{eff}}^{2},} \\
\varepsilon_{\mathrm{i}}=\varepsilon_{0}\left(1-\frac{\omega_{\mathrm{pi}}^{2}}{\omega^{2}+v_{\mathrm{eff}}^{2}}\right) .
\end{gathered}
$$

In the above equations, $\omega_{\mathrm{pi}}$ is the ion-plasma frequency. For convenience, $v(u)$ is replaced by the effective collision frequency $\left(v_{\text {eff }}=v_{\text {en }}+v_{\text {ei }}+v_{\text {ed }}\right)$. The effective collision frequency is the sum of electron-neutral, electron-ion and electron-dust particle collision frequencies. However, in this study the effective collision frequency for $\sigma_{\mathrm{i}}$ and $\varepsilon_{\mathrm{i}}$ refers to the collisions of ions with neutral particles $\left(v_{\text {eff }}=v_{\text {in }}\right)$.

\section{Effect of the Charging Process}

The direct collisions of electrons or ions with charged dust particles are often referred to as the charging process of dust particles. In dusty plasmas, the dust charging process by electrons and ions is also different from that of ion-electron plasmas. Before the incident EM wave strikes the dusty plasma, the dust particles are in equilibrium with charge $q_{\mathrm{do}}$. At equilibrium state, the ion and electron currents are equal, so no current is flowing onto the surface of dust particles. However, when the incident wave strikes the dusty plasma, the external electric field destroys the original equilibrium by changing the charge on dust particles from $q_{\mathrm{d} 0}$ to $q_{\mathrm{d} 0}+q_{\mathrm{d} 1}$ (Jia JS et al., 2015). The charging equation of the dust particles is given as (Shukla and Mamun, 2002):

$$
\frac{\partial q_{\mathrm{d} 1}}{\partial t}+v_{\mathrm{ch}} q_{\mathrm{d} 1}=l_{\mathrm{e} 1}+l_{\mathrm{i} 1}
$$

where $v_{\mathrm{ch}}$ is the charging frequency (charge relaxation rate), and $I_{\mathrm{e} 1}$ and $I_{\mathrm{i} 1}$ are the electron and ion charging currents, respectively (Jana et al., 1993). Charging current is related to the number density of electrons and ions, quantity of charge, and temperature.
Equation (9) can be written as:

$$
\left(\mathrm{i} \omega+v_{\mathrm{ch}}\right) q_{\mathrm{d} 1}=l_{\mathrm{e} 1}+l_{\mathrm{i} 1} .
$$

The charging current and electric field are related by the following equation:

$$
j_{\mathrm{d} 1}=\sigma_{\mathrm{d} 1 \text { complex }} E=\left(\sigma_{\mathrm{d} 1}+\mathrm{i} \omega \varepsilon_{0} X \mathrm{~d} 1\right) E .
$$

Considering the external EM field is:

$$
E=E_{0} \mathrm{e}^{\mathrm{i} \omega t-\mathrm{i} k \cdot r}
$$

according to the orbit-limited motion (OLM) approach (Shukla and Mamun, 2002):

$$
N_{\mathrm{d}} q_{\mathrm{d} 1}=\frac{N_{\mathrm{d}}}{\left(\mathrm{i} \omega+v_{\mathrm{ch}}\right)}\left(l_{\mathrm{e} 1}+l_{\mathrm{i} 1}\right)=\rho_{\mathrm{d} 1}
$$

where $\rho_{\mathrm{d} 1}$ is the charge density. Consider the Maxwell equation:

$$
\nabla \cdot j_{\mathrm{d} 1}=\frac{\partial}{\partial t} \nabla \cdot D=-\frac{\partial \rho_{\mathrm{d} 1}}{\partial t}
$$

where $j_{\mathrm{d} 1}$ is the charging current of electrons and ions which adds the charge $q_{\mathrm{d} 1}$ on a dust particle. The number of electrons and ions determines the charge on a dust particle, and it is assumed that the size and charge of each dust particle are the same. Using Equations (11), (12) and (13) into (14) we then obtain:

$$
\left(\sigma_{\mathrm{d} 1}+\mathrm{i} \omega \varepsilon_{0} X \mathrm{~d} 1\right) k E=\frac{\omega N_{\mathrm{d}}}{\left(\mathrm{i} \omega+v_{\mathrm{ch}}\right)}\left(l_{\mathrm{e} 1}+l_{\mathrm{i} 1}\right) .
$$

We further assume that the velocity distribution of the plasma species is Maxwellian. The electron and ion charging currents caused by the electric field are given as (Ma JX and Yu MY, 1994):

$$
\begin{aligned}
& l_{\mathrm{e} 1}=-e \int_{v_{\mathrm{m}}}^{\infty} v \sigma_{\mathrm{e}}^{\mathrm{d}} f_{\mathrm{e}} \mathrm{d} V=-\frac{8 \mathrm{e}^{2} N_{\mathrm{e}} E}{3 \sqrt{\pi} m_{\mathrm{e}}} \int_{u_{\mathrm{m}}}^{\infty} \frac{\sigma_{\mathrm{e}}^{\mathrm{d}} u^{4} \mathrm{e}^{-u^{2}} \mathrm{~d} u}{\mathrm{i} \omega+v_{\mathrm{e}}(u)}, \\
& l_{\mathrm{i} 1}=e \int_{0}^{\infty} v \sigma_{\mathrm{i}}^{\mathrm{d}} f_{\mathrm{i}} \mathrm{d} V=-\frac{8 \mathrm{e}^{2} N_{\mathrm{i}} E}{3 \sqrt{\pi} m_{\mathrm{i}}} \int_{0}^{\infty} \frac{\sigma_{\mathrm{i}}^{\mathrm{d}} u^{4} \mathrm{e}^{-u^{2}} \mathrm{~d} u}{\mathrm{i} \omega+v_{\mathrm{i}}(u)}
\end{aligned}
$$

Substituting the solution of Equations (16) and (17) into Equation (15), we obtain the following equations:

$$
\begin{aligned}
\left(\sigma_{\mathrm{d} 1}+\mathrm{i} \omega \varepsilon_{0} X \mathrm{~d} 1\right) k E= & -\frac{\omega N_{\mathrm{d}}}{\left(\mathrm{i} \omega+v_{\mathrm{ch}}\right)} \frac{8 \mathrm{e}^{2} N_{\mathrm{e}} E}{3 \sqrt{\pi} m_{\mathrm{e}}} \int_{u_{\mathrm{m}}}^{\infty} \frac{u^{4} \mathrm{e}^{-u^{2}} \sigma_{\mathrm{e}}^{\mathrm{d}} \mathrm{d} u}{\mathrm{i} \omega+v_{\mathrm{e}}(u)} \\
& -\frac{\omega N_{\mathrm{d}}}{\left(\mathrm{i} \omega+v_{\mathrm{ch}}\right)} \frac{8 \mathrm{e}^{2} N_{\mathrm{i}} E}{3 \sqrt{\pi} m_{\mathrm{i}}} \int_{0}^{\infty} \frac{u^{4} \mathrm{e}^{-u^{2}} \sigma_{\mathrm{i}}^{\mathrm{d}} \mathrm{d} u}{\mathrm{i} \omega+v_{\mathrm{i}}(u)}
\end{aligned}
$$

where $u_{\mathrm{m}}$ is the minimum value of electron velocity at which the electron hits the dust particle, $\sigma_{\mathrm{e}}^{\mathrm{d}}$ and $\sigma_{\mathrm{i}}^{\mathrm{d}}$ are the collision crosssections of charging given as:

$$
\begin{aligned}
& \sigma_{\mathrm{e}}^{\mathrm{d}}=\pi r_{\mathrm{d}}^{2}\left(1-\frac{2 e \phi_{\mathrm{d}}}{m_{\mathrm{e}} v_{\mathrm{e}}^{2}}\right), \\
& \sigma_{\mathrm{i}}^{\mathrm{d}}=\pi r_{\mathrm{d}}^{2}\left(1+\frac{2 e \phi_{\mathrm{d}}}{m_{\mathrm{i}} v_{\mathrm{i}}^{2}}\right) .
\end{aligned}
$$

Here $r_{\mathrm{d}}$ is the dust radius (a spherical dust particle is assumed), and $\phi_{\mathrm{d}}$ is the potential difference between the particle and background plasma. If $2 e \phi_{\mathrm{d}} / m_{\mathrm{e}} v_{\mathrm{e}}^{2}$ and $2 e \phi_{\mathrm{d}} / m_{\mathrm{i}} v_{\mathrm{i}}^{2}$ are $\ll 1$ then collision cross-sections of charging $\sigma_{\mathrm{e}}^{\mathrm{d}}=\sigma_{\mathrm{i}}^{\mathrm{d}}=\pi r_{\mathrm{d}}^{2}$ and $u_{\mathrm{m}}=0$. The real and imaginary parts in both sides of the Equation (18) are equal and can be written as: 


$$
\begin{gathered}
\sigma_{\mathrm{d} 1}=\eta_{\mathrm{ed}} \frac{\left(\omega^{2}-v_{\mathrm{ch}} v_{\text {eff }}\right)}{\left(\omega^{2}+v_{\text {eff }}^{2}\right)\left(\omega^{2}+v_{\mathrm{ch}}^{2}\right)} v_{\phi}+\eta_{\mathrm{id}} \frac{\left(\omega^{2}-v_{\mathrm{ch}} v_{\text {eff }}\right)}{\left(\omega^{2}+v_{\text {eff }}^{2}\right)\left(\omega^{2}+v_{\mathrm{ch}}^{2}\right)} v_{\phi \prime} \quad(21) \\
\varepsilon_{\mathrm{d} 11}=\varepsilon_{0} X_{\mathrm{d} 11}=\eta_{\mathrm{ed}} \frac{v_{\mathrm{ch}}+v_{\mathrm{eff}}}{\left(\omega^{2}+v_{\mathrm{eff}}^{2}\right)\left(\omega^{2}+v_{\mathrm{ch}}^{2}\right)} v_{\phi}+\eta_{\mathrm{id}} \frac{v_{\mathrm{ch}}+v_{\mathrm{eff}}}{\left(\omega^{2}+v_{\mathrm{eff}}^{2}\right)\left(\omega^{2}+v_{\mathrm{ch}}^{2}\right)} v_{\phi \prime}
\end{gathered}
$$

where $\eta_{j \mathrm{~d}}=e^{2} \pi r_{\mathrm{d}}^{2} N_{j} N_{\mathrm{d}} / m_{j}$ is the charging response factor for species $j$ and $v_{\phi}=\omega / k$ is the phase velocity of radio waves in weaklyionized dusty plasmas which is equal to the speed of light. Now, the complex conductivity and permittivity of the weakly ionized dusty plasma containing both the collision and charging effects can be written as:

$$
\begin{aligned}
\sigma_{\mathrm{c}}= & \sigma_{\mathrm{e}}+\sigma_{\mathrm{i}}+\sigma_{\mathrm{d} 1}=\varepsilon_{0} \frac{\omega_{\mathrm{pe}}^{2} v_{\mathrm{eff}}}{\omega^{2}+v_{\mathrm{eff}}^{2}}+\varepsilon_{0} \frac{\omega_{\mathrm{pi}}^{2} v_{\mathrm{eff}}}{\omega^{2}+v_{\mathrm{eff}}^{2}} \\
& \left.+\eta_{\mathrm{ed}} \frac{\left(\omega^{2}-v_{\mathrm{ch}} v_{\mathrm{eff}}\right)}{\left(\omega^{2}+v_{\mathrm{eff}}^{2}\right)\left(\omega^{2}+v_{\mathrm{ch}}^{2}\right)} v_{\phi}+\eta_{\mathrm{id}} \frac{\left(\omega^{2}-v_{\mathrm{ch}} v_{\text {eff }}\right)}{\left(\omega^{2}+v_{\mathrm{eff}}^{2}\right)\left(\omega^{2}+v_{\mathrm{ch}}^{2}\right)} v_{\phi}\right\}, \\
\varepsilon_{\mathrm{c}}= & \varepsilon_{\mathrm{e}}+\varepsilon_{\mathrm{i}}+\varepsilon_{\mathrm{d} 1}=\varepsilon_{0}\left(1-\frac{\omega_{\mathrm{pe}}^{2}}{\omega^{2}+v_{\mathrm{eff}}^{2}}-\frac{\omega_{\mathrm{pi}}^{2}}{\omega^{2}+v_{\mathrm{eff}}^{2}}\right) \\
& \left.+\eta_{\mathrm{ed}} \frac{v_{\mathrm{ch}}+v_{\mathrm{eff}}}{\left(\omega^{2}+v_{\mathrm{eff}}^{2}\right)\left(\omega^{2}+v_{\mathrm{ch}}^{2}\right)} v_{\phi}+\eta_{\mathrm{id}} \frac{v_{\mathrm{ch}}+v_{\mathrm{eff}}}{\left(\omega^{2}+v_{\mathrm{eff}}^{2}\right)\left(\omega^{2}+v_{\mathrm{ch}}^{2}\right)} v_{\phi}\right\} .
\end{aligned}
$$

Neglecting the effect of ions, Equations (23) and (24) can be written as:

$$
\begin{aligned}
\sigma_{\mathrm{c}} & =\sigma_{\mathrm{e}}+\sigma_{\mathrm{d} 1} \\
& \left.=\varepsilon_{0} \frac{\omega_{\mathrm{pe}}^{2} v_{\mathrm{eff}}}{\omega^{2}+v_{\mathrm{eff}}^{2}}+\eta_{\mathrm{ed}} \frac{\left(\omega^{2}-v_{\mathrm{ch}} v_{\mathrm{eff}}\right)}{\left(\omega^{2}+v_{\mathrm{eff}}^{2}\right)\left(\omega^{2}+v_{\mathrm{ch}}^{2}\right)} v_{\phi}\right\}, \\
\varepsilon_{\mathrm{c}}= & \varepsilon_{\mathrm{e}}+\varepsilon_{\mathrm{d} 1} \\
= & \left.\varepsilon_{0}\left(1-\frac{\omega_{\mathrm{pe}}^{2}}{\omega^{2}+v_{\mathrm{eff}}^{2}}\right)+\eta_{\mathrm{ed}} \frac{v_{\mathrm{ch}}+v_{\mathrm{eff}}}{\left(\omega^{2}+v_{\mathrm{eff}}^{2}\right)\left(\omega^{2}+v_{\mathrm{ch}}^{2}\right)} v_{\phi}\right\} .
\end{aligned}
$$

Equations (25) and (26) are used in common dusty plasmas where the effect of ions is not considered.

\section{Numerical Results}

In the mesosphere, the plasma and dust parameters between 80 and $90 \mathrm{~km}$ were set as: the mass of an electron, $m_{\mathrm{e}}=9.1 \times 10^{-31}$ $\mathrm{kg}$; the mass of an ion, $m_{\mathrm{i}}=50 \times m_{\mathrm{p}}=8.3631 \times 10^{-26} \mathrm{~kg}$, where $m_{\mathrm{p}}$ is the mass of a proton; electron charge, $e=1.6 \times 10^{-19} \mathrm{C}$; and neutral density, $N_{\mathrm{n}}=1 \times 10^{20} \mathrm{~m}^{-3}$. Phase velocity of radio waves $v_{\phi}=\omega / k=C$ where $C$ is the speed of light equal to $3 \times 10^{8} \mathrm{~m} / \mathrm{s}$. Further detail of mesospheric parameters is shown in Table 1. Note that in Table 1, case 1 corresponds to Equations (23) and (24) where the ion effect is considered. In contrast, case 2 corresponds to Equations (25) and (26) where the ion effect is not considered.

In the mesosphere, the typical values of dust and plasma densities are $N_{\mathrm{d}}=N_{\mathrm{e}}=N_{\mathrm{i}}=10^{9} \mathrm{~m}^{-3}$ (Mahmoudian and Scales, 2012). However, at PMSE altitudes, ion, electron and dust densities vary, which gives rise to different mesospheric conditions, including the bite-out condition. During the summer months at polar latitude, the temperature drops, reaching a mean summer minimum temperature of $\sim 130 \mathrm{~K}$. This extremely low temperature allows the dust particles to form at altitudes between 80 and $90 \mathrm{~km}$ and thus increases the dust number density at PMSE altitudes (Rapp and Lübken, 2004; Scales and Mahmoudian, 2016). In favorable conditions, the dust size grows ranging from $\sim 5-50 \mathrm{~nm}$ (Rapp and Lübken, 2004; Routledge et al., 2011). Free electrons from surrounding plasma attach to these dust particles and hence a local reduction in free electron density is produced which is called the electron bite-out. Increasing the dust number density and dust size strengthens the bite-out. The presence of dust is necessary for the bite-out condition, however, it has no threshold of dust density at which it occurs necessarily. At different PMSE altitudes, it occurs at different dust density; for example, at one height it may occur at $N_{\mathrm{d}}=10^{10} \mathrm{~m}^{-3}$, then at another height, it is not necessary that there should be a bite-out at the same dust density. At another height, the bite-out may occur at a smaller dust density than $N_{d}=10^{10} \mathrm{~m}^{-3}$ or maybe occurs at a higher dust density than $N_{\mathrm{d}}=10^{10} \mathrm{~m}^{-3}$. In any case, the dust density should be high to sink enough free electrons to produce a reduction in free electron density, and hence make $N_{\mathrm{e}} \ll N_{\mathrm{i}}$.

\subsection{Complex Conductivity Without the Bite-Out Condition}

Figure 1 shows the effect of different mesospheric parameters and EM wave frequency on complex conductivity without the electron bite-out condition, for cases 1 and 2 as shown in Table 1.
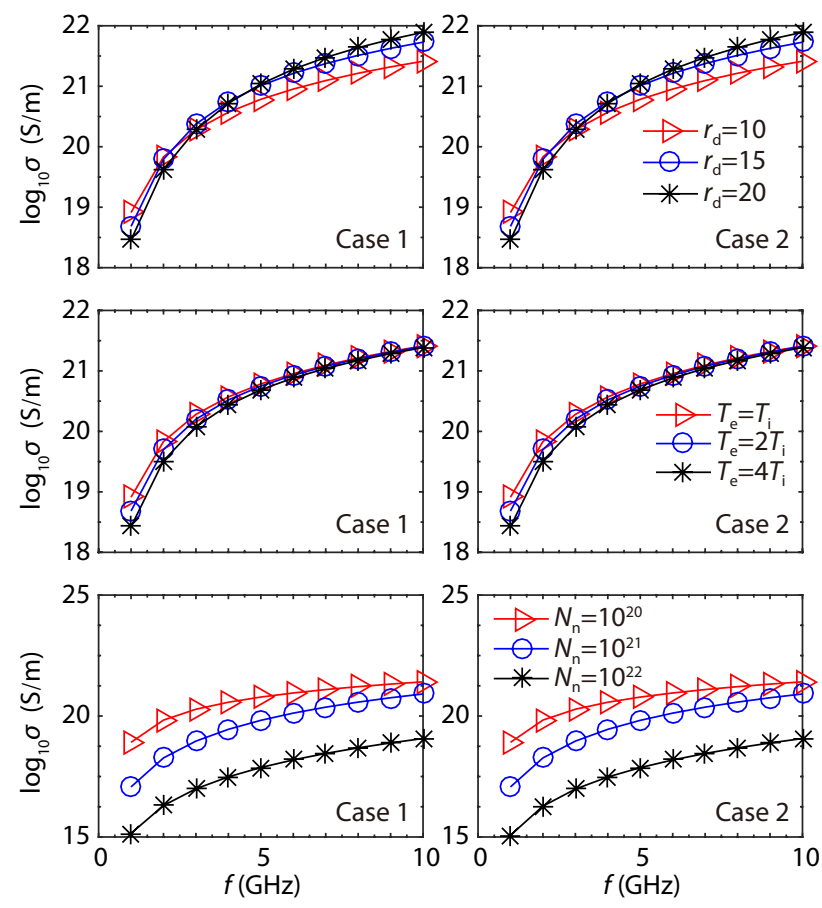

Figure 1. The effect of different parameters on complex conductivity without the bite-out condition $\left(N_{\mathrm{e}} \approx N_{\mathrm{i}}\right)$ of Table 1. Left panels: corresponds to case 1 of Table 1; right panels: corresponds to case 2 of Table 1. First row: shows the effect of dust size on conductivity. Second row: shows the effect of enhanced electron temperature due to HF heating on conductivity. The unheated background electron temperature is assumed as $T_{\mathrm{e}}=T_{\mathrm{i}}=150 \mathrm{~K}$. Third row: shows the effect of neutral particle density on conductivity. The legends are shown at the right side of each row. Dust size is $\mathrm{nm}$, temperature is in $\mathrm{K}$, and neutral density is in $\mathrm{m}^{-3}$. 
Table 1. Details of different mesospheric conditions and cases.

\begin{tabular}{ccccc}
\hline Conditions & $N_{\mathrm{e}}\left(\mathrm{m}^{-3}\right)$ & $N_{\mathrm{d}}\left(\mathrm{m}^{-3}\right)$ & $N_{\mathrm{i}}\left(\mathrm{m}^{-3}\right)$ & $N_{\mathrm{e}} / N_{\mathrm{i}}$ \\
\hline without bite-out & $1 \times 10^{10}$ & $1 \times 10^{6}$ & $1.0001 \times 10^{10}$ & $N_{\mathrm{e}} \approx N_{\mathrm{i}}$ \\
bite-out & $1 \times 10^{6}$ & $1 \times 10^{10}$ & $1.0001 \times 10^{10}$ & $N_{\mathrm{e}} \ll N_{\mathrm{i}}$
\end{tabular}

Case 1: corresponds to Equations (23) and (24)

Case 2: corresponds to Equations (25) and (26)

The first row of Figure 1 presents the numerical results for different dust sizes. It is clear that in both cases complex conductivity increases with increasing wave frequency and dust size. The second row presents the results for different values of electron temperatures. Before the HF heater switched on, the pre-heated electron temperature is assumed to be equal to neutral or ion temperature $T_{\mathrm{n}}=T_{\mathrm{e}}=T_{\mathrm{i}}=150 \mathrm{~K}$ (Kassa et al., 2005). The enhanced electron temperatures due to heating were assumed as $T_{\mathrm{e}}=2 T_{\mathrm{i}}$ and $T_{\mathrm{e}}=4 T_{\mathrm{i}}$. It is clear that in both cases, complex conductivity decreases with increasing electron temperature for the frequency range $f \leq 4 \mathrm{GHz}$, however for comparatively larger frequencies ( $f \geq$ $5 \mathrm{GHz}$ ), the conductivity for different values of electron temperatures shows no variation. The third row of Figure 1 indicates that in cases 1 and 2, conductivity decreases with increasing neutral particle density.

\subsection{Complex Conductivity in the Bite-Out Condition}

Similar to Figure 1, Figure 2 shows the effect of different parameters on complex conductivity but for the electron bite-out condition of Table 1. For the bite-out condition, the dependence of conductivity on dust size, electron temperature and neutral density shows similar behavior as the absent bite-out condition of Figure 1. However, unlike Figure 1, the results of cases 1 and 2 are different
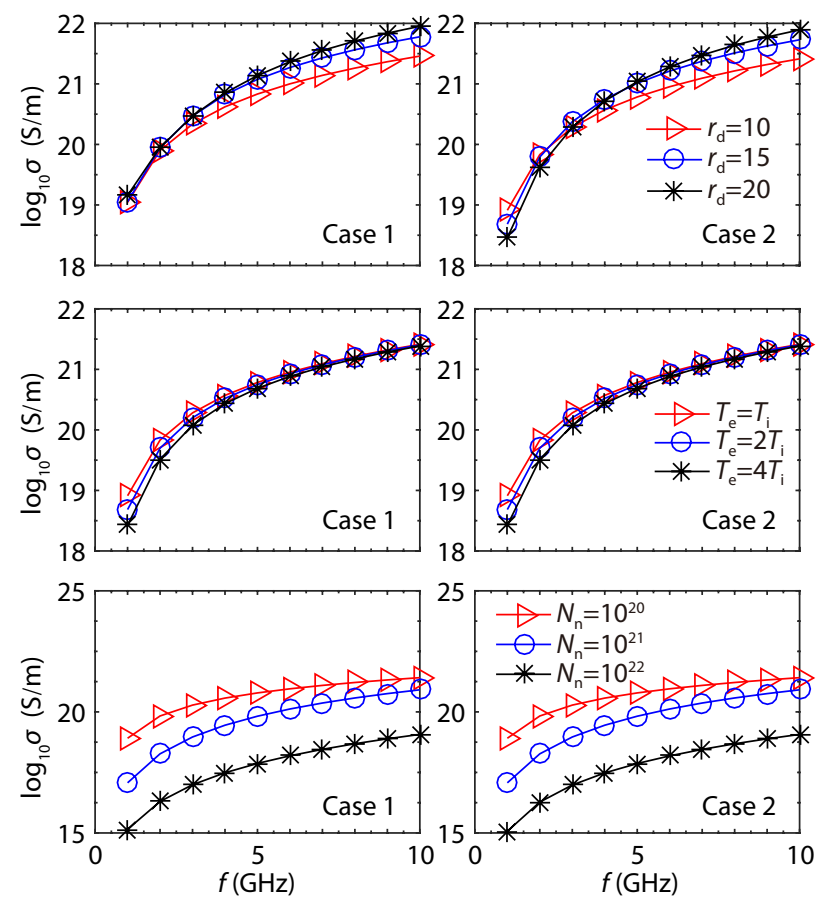

Figure 2. As for Figure 1 but for electron bite-out condition $\left(N_{\mathrm{e}} \ll N_{\mathrm{i}}\right)$ of Table 1 . in the first row for the frequency range $f \leq 2 \mathrm{GHz}$. The increase in charging response factor of ion $\left(\eta_{\mathrm{id}}\right)$ and plasma-ion frequency $\left(\omega_{\text {pi }}\right)$ due to high dust density and the increase in effective collision frequency of ions ( $\left.v_{\text {eff_ion }}\right)$ due to increase in dust size makes the difference in the results of cases 1 and 2 . In the second and third rows, the results between cases 1 and 2 show no clear difference.

\subsection{Complex Conductivity Dependence on $\boldsymbol{N}_{\mathrm{d}} / \boldsymbol{N}_{\mathrm{e}}$}

In both mesospheric conditions of Table 1, the densities of electrons, dust and ions are constant. Figure 3 shows the dependence of complex conductivity on different parameters for the dust to electron density ratio. Here, we consider the quiet condition when there is no electron precipitation, and thus no free electrons will reach the PMSE altitudes between $80-90 \mathrm{~km}$, consequently, the electron density will remain constant as $N_{\mathrm{e}}=1 \times 10^{5} \mathrm{~m}^{-3}$. For increasing dust density $\left(N_{\mathrm{d}}\right)$ the values of ion densities were calculated and are shown in Table 2 ; it is clear that for constant $N_{\mathrm{e}}$ the ratio $N_{\mathrm{d}} / N_{\mathrm{e}}$ and $N_{\mathrm{i}}$ increases with increasing $N_{\mathrm{d}}$, consequently $N_{\mathrm{e}} / N_{\mathrm{i}}$ decreases. In Figure 3, at any value of $N_{\mathrm{d}} / N_{\mathrm{e}}$ when the divergence arises between the results of cases 1 and 2, it means that at this value and onward the effect of ions on complex conductivity is now significant and cannot be ignored. The first and second rows of Figure 3 indicate that in cases 1 and 2, for both dust sizes (10 and $20 \mathrm{~nm}$ ) and electron temperatures $\left(T_{\mathrm{e}}=T_{\mathrm{i}}\right.$ and $T_{\mathrm{e}}=4 T_{\mathrm{i}}$ ), complex conductivity increases with increasing $N_{\mathrm{d}} / N_{\mathrm{e}}$. Furthermore, in case 1 the complex conductivity is greater than that in case 2 . The difference between cases 1 and 2 appears early for larger dust size $(20 \mathrm{~nm})$ and higher electron temperature $\left(T_{\mathrm{e}}=4 T_{\mathrm{i}}\right)$. It is clear that for smaller dust size $(10 \mathrm{~nm})$ and low electron temperature $\left(T_{\mathrm{e}}=T_{\mathrm{i}}\right)$ at ratio $N_{\mathrm{d}} / N_{\mathrm{e}} \geq 10^{5}$, there is a difference between the results of cases 1 and 2. Table 2 indicates that for ratio $N_{\mathrm{d}} / N_{\mathrm{e}}=$ $10^{5}$, the corresponding $N_{\mathrm{e}} / N_{\mathrm{i}}=0.99 \times 10^{-5}$. On the other hand, for
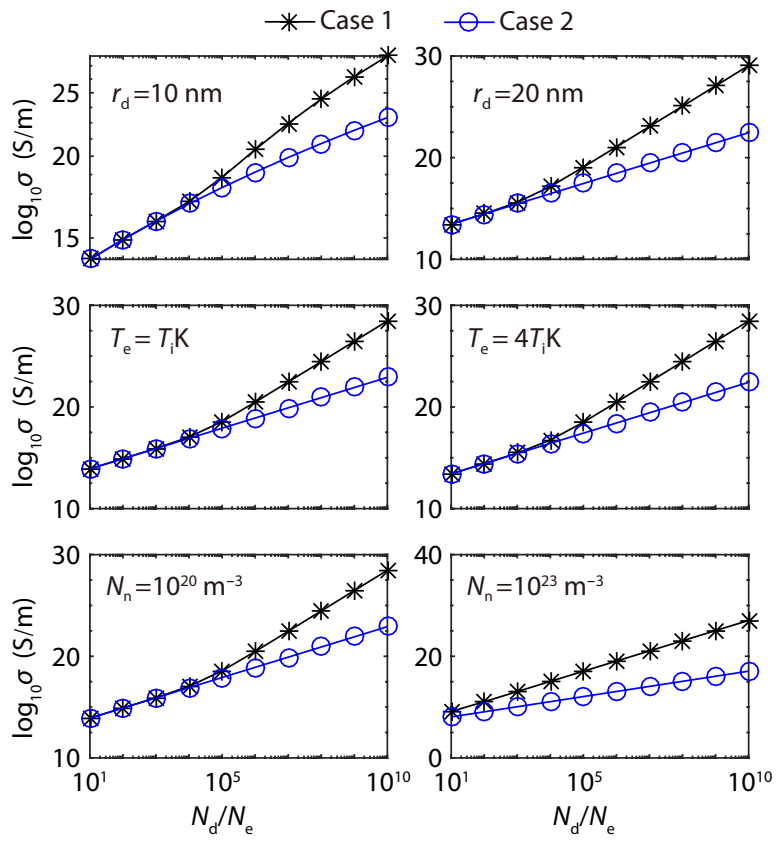

Figure 3. Dependence of complex conductivity on dust-to-electron density ratio $\left(N_{\mathrm{d}} / N_{\mathrm{e}}\right)$ for different parameters and cases. 
Table 2. Numerical values of $N_{\mathrm{e}}, N_{\mathrm{d}}$ and $N_{\mathrm{i}}$ used in Figures 3 and 6 .

\begin{tabular}{cccc}
\hline$N_{\mathrm{e}}\left(\mathrm{m}^{-3}\right)$ & $N_{\mathrm{d}} / N_{\mathrm{e}}$ & $N_{\mathrm{i}}\left(\mathrm{m}^{-3}\right)$ & $N_{\mathrm{e}} / N_{\mathrm{i}}$ \\
\hline $1 \times 10^{5}$ & $10^{1}$ & $1.1 \times 10^{6}$ & $0.90 \times 10^{-1}$ \\
$1 \times 10^{5}$ & $10^{2}$ & $1.01 \times 10^{7}$ & $0.99 \times 10^{-2}$ \\
$1 \times 10^{5}$ & $10^{3}$ & $1.001 \times 10^{8}$ & $0.99 \times 10^{-3}$ \\
$1 \times 10^{5}$ & $10^{4}$ & $1.0001 \times 10^{9}$ & $0.99 \times 10^{-4}$ \\
$1 \times 10^{5}$ & $10^{5}$ & $1.00001 \times 10^{10}$ & $0.99 \times 10^{-5}$ \\
$1 \times 10^{5}$ & $10^{6}$ & $1.000001 \times 10^{11}$ & $0.99 \times 10^{-6}$ \\
$1 \times 10^{5}$ & $10^{7}$ & $1.0000001 \times 10^{12}$ & $0.99 \times 10^{-7}$ \\
$1 \times 10^{5}$ & $10^{8}$ & $1.00000001 \times 10^{13}$ & $0.99 \times 10^{-8}$ \\
$1 \times 10^{5}$ & $10^{9}$ & $1.000000001 \times 10^{14}$ & $0.99 \times 10^{-9}$ \\
$1 \times 10^{5}$ & $10^{10}$ & $1.0000000001 \times 10^{15}$ & $0.99 \times 10^{-10}$ \\
\hline
\end{tabular}

larger dust size $(20 \mathrm{~nm})$ and higher electron temperature $\left(T_{\mathrm{e}}=4 T_{\mathrm{i}}\right)$, the difference between the results of cases 1 and 2 starts at ratio $N_{\mathrm{d}} / N_{\mathrm{e}} \geq 10^{4}$. Table 2 shows that for ratio $N_{\mathrm{d}} / N_{\mathrm{e}}=10^{4}$ the corresponding $N_{\mathrm{e}} / N_{\mathrm{i}}=0.99 \times 10^{-4}$. This means that for smaller dust size and low electron temperature at the comparatively smaller value of ratio $N_{\mathrm{e}} / N_{\mathrm{i}}\left(0.99 \times 10^{-5}\right)$, and for larger dust size and high electron temperature at the comparatively greater value of ratio $N_{\mathrm{e}} / N_{\mathrm{i}}$ $\left(0.99 \times 10^{-4}\right)$, the effect of ions for complex conductivity becomes significant. Row 3 indicates that for both cases at neutral densities $10^{20} \mathrm{~m}^{-3}$ and $10^{23} \mathrm{~m}^{-3}$, conductivity increases with increasing ra-
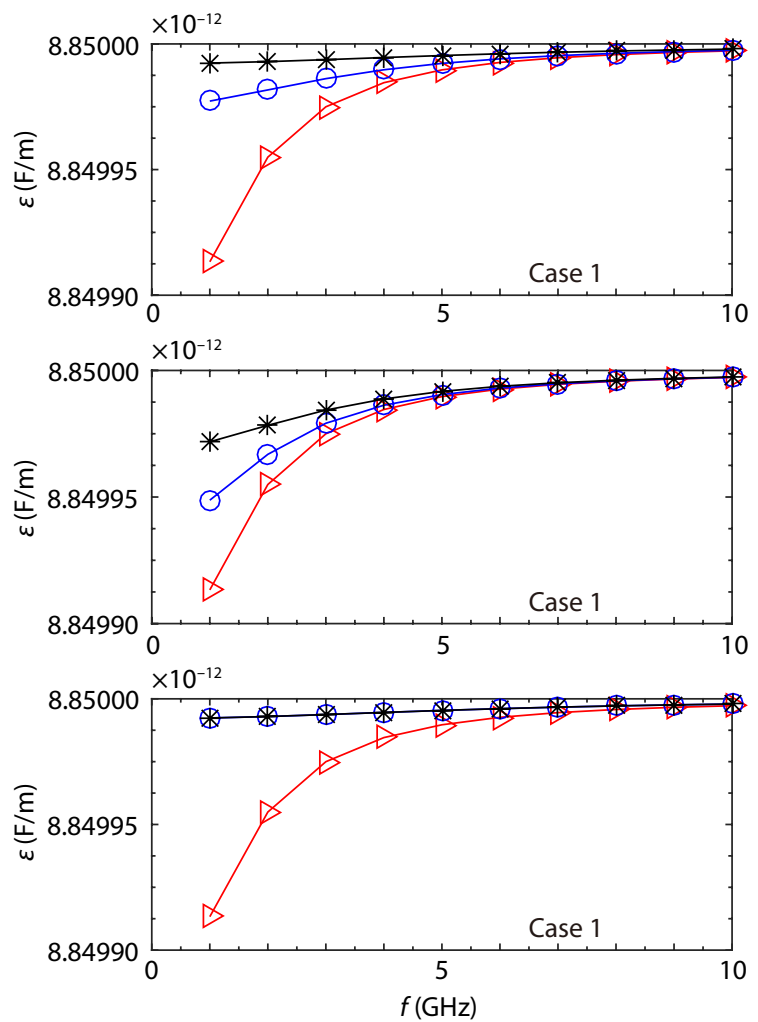

tio $N_{\mathrm{d}} / N_{\mathrm{e}}$. For both neutral densities in case 1 , conductivity is greater than that in case 2 . It is clear that for lower neutral density $\left(10^{20} \mathrm{~m}^{-3}\right)$ there are growing differences between the results of cases 1 and 2 at ratio $N_{\mathrm{d}} / N_{\mathrm{e}} \geq 10^{5}$. Table 2 indicates that for $N_{\mathrm{d}} / N_{\mathrm{e}}=$ $10^{5}$ the corresponding value of ratio $N_{\mathrm{e}} / N_{\mathrm{i}}$ is $0.99 \times 10^{-5}$. Whereas for higher neutral density $\left(10^{23} \mathrm{~m}^{-3}\right)$, the divergence arises between the results of cases 1 and 2 at ratio $N_{\mathrm{d}} / N_{\mathrm{e}} \geq 10^{2}$. From Table 2 it is clear that for ratio $N_{\mathrm{d}} / N_{\mathrm{e}}=10^{2}$ the corresponding value of ratio $N_{\mathrm{e}} / N_{\mathrm{i}}$ is $0.99 \times 10^{-2}$. This means that for low neutral density at the comparatively smaller value of ratio $N_{\mathrm{e}} / N_{\mathrm{i}}(0.99 \times$ $\left.10^{-5}\right)$, and for high neutral density at the comparatively greater value of ratio $N_{\mathrm{e}} / N_{\mathrm{i}}\left(0.99 \times 10^{-2}\right)$, the effect of ions on complex conductivity can be considered.

\subsection{Complex Permittivity Without the Bite-Out Condition}

Figure 4 shows the effect of different parameters and EM wave frequency on complex permittivity without the electron bite-out condition for cases 1 and 2 as shown in Table 1. Figure 4 indicates that in cases 1 and 2, the complex permittivity increases with increasing dust size, electron temperature and neutral density. Furthermore, it is clear that for each parameter, the increase in complex permittivity is smaller than the permittivity of free space $\left(\varepsilon_{\mathrm{c}}<\varepsilon_{0}\right)$.

\subsection{Complex Permittivity in Bite-Out Condition}

As in Figure 4, Figure 5 shows the effect of different mesospheric parameters on $\varepsilon$ but for the electron bite-out condition of Table 1.
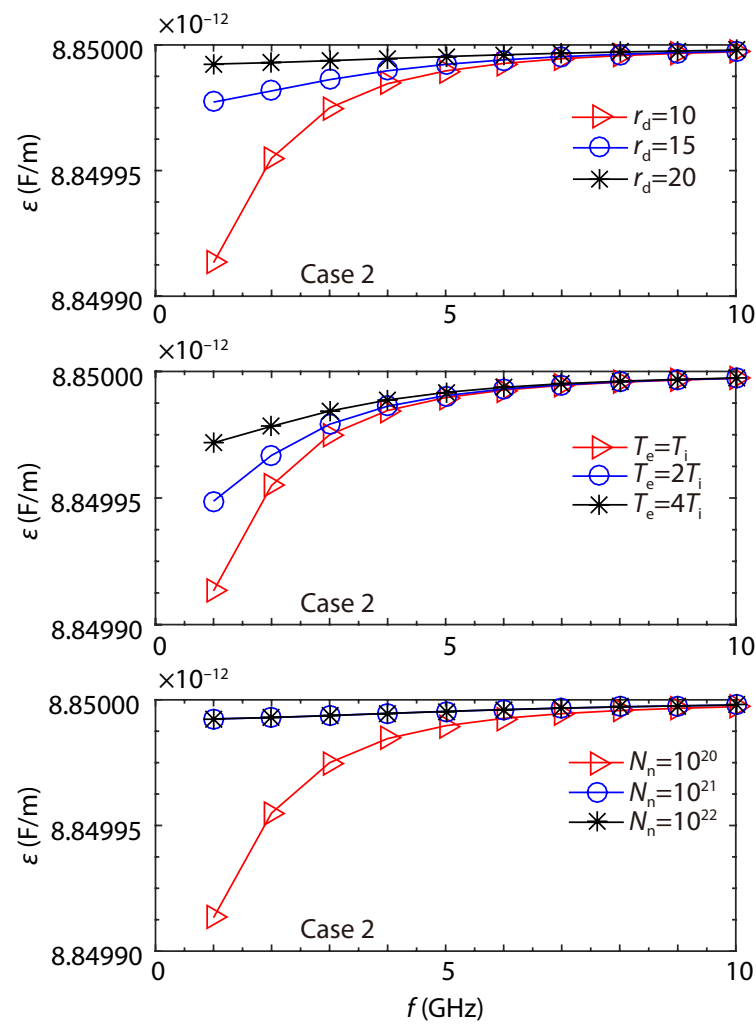

Figure 4. The effect of different parameters on complex permittivity without the bite-out condition $\left(N_{\mathrm{e}} \approx N_{\mathrm{i}}\right)$ of Table 1. Left panels: correspond to case 1 of Table 1. Right panels: correspond to case 2 of Table 1. First row: shows the effect of dust size on $\varepsilon$. Second row: shows the effect of enhanced electron temperature due to HF heating on $\varepsilon$. Third row: shows the effect of neutral particle density on $\varepsilon$. The legends are shown at the right side of each row. Dust size is $\mathrm{nm}$, temperature is in $\mathrm{K}$, and neutral density is in $\mathrm{m}^{-3}$. 

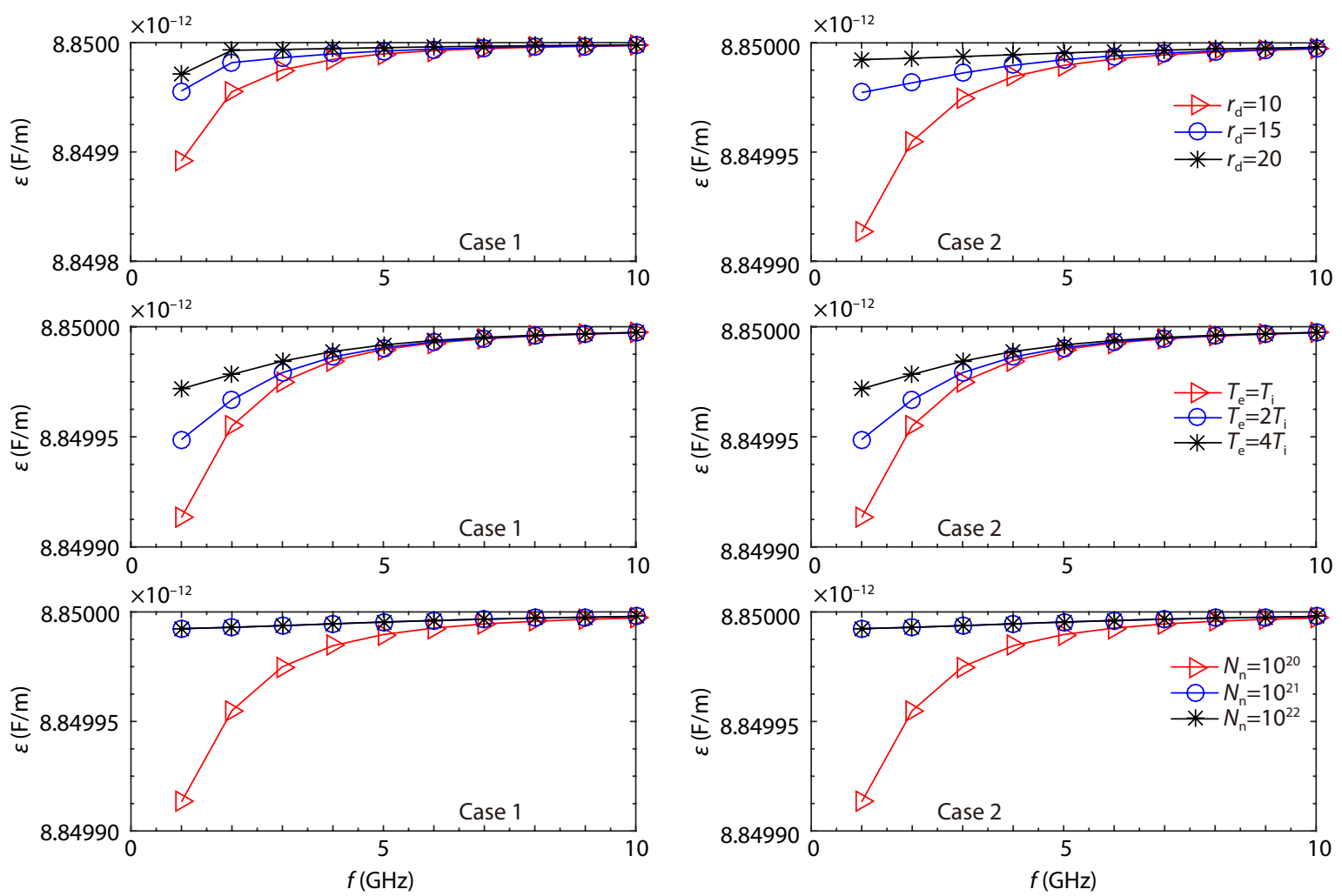

Figure 5. Same as for Figure 4 but for electron bite-out condition $\left(N_{e} \ll N_{i}\right)$ from Table 1 .

For the bite-out condition, the results shown in Figure 5 are similar to those shown in Figure 4. However, unlike Figure 4, the results of cases 1 and 2 are different. In the second and third rows, the results of cases 1 and 2 show no clear difference.

\subsection{Complex Permittivity Dependence on $\boldsymbol{N}_{\mathrm{d}} / \mathbf{N}_{\mathbf{e}}$}

Figure 6 shows the dependence of complex permittivity using the same parameters as in Figure 3. The first and second rows indicate that for both values of dust sizes (10 and $20 \mathrm{~nm}$ ) and electron temperatures $\left(T_{\mathrm{e}}=T_{\mathrm{i}}\right.$ and $\left.T_{\mathrm{e}}=4 T_{\mathrm{i}}\right)$, complex permittivity in case 2 shows no variation with increasing ratio $N_{\mathrm{d}} / N_{\mathrm{e}}$, however, for $N_{\mathrm{d}} / N_{\mathrm{e}} \geq 10^{9}$ it decreases in case 1 . For both values of dust sizes and electron temperatures, the difference between the results of cases 1 and 2 start at ratio $N_{\mathrm{d}} / N_{\mathrm{e}} \geq 10^{9}$. This means that at PMSE altitudes between 80 to $90 \mathrm{~km}$ at ratio $N_{\mathrm{d}} / N_{\mathrm{e}} \geq 10^{9}$, the ion role becomes significant.

The third row of Figure 6 indicates that in case 2, for both neutral densities $\left(10^{20}\right.$ and $\left.10^{23} \mathrm{~m}^{-3}\right)$, the complex permittivity shows no variation with increasing $N_{\mathrm{d}} / N_{\mathrm{e}}$. However, for both neutral densities in case 1 , the complex permittivity decreases. It is clear that for low neutral density $\left(10^{20} \mathrm{~m}^{-3}\right)$ there is a divergence between the results of cases 1 and 2 at ratio $N_{\mathrm{d}} / N_{\mathrm{e}} \geq 10^{9}$. Whereas for high neutral density $\left(10^{23} \mathrm{~m}^{-3}\right)$, the divergence between the results of cases 1 and 2 at ratio $N_{\mathrm{d}} / N_{\mathrm{e}} \geq 10^{10}$. As from Table 2 it is clear that for $N_{\mathrm{d}} / N_{\mathrm{e}}=10^{9}$ the corresponding $N_{\mathrm{e}} / N_{\mathrm{i}}=0.99 \times 10^{-9}$ and for $N_{\mathrm{d}} / N_{\mathrm{e}}=10^{10}$ the corresponding $N_{\mathrm{e}} / N_{\mathrm{i}}=0.99 \times 10^{-10}$. This means that for low neutral density $\left(10^{20} \mathrm{~m}^{-3}\right)$ at comparatively greater values of ratio $N_{\mathrm{e}} / N_{\mathrm{i}}\left(0.99 \times 10^{-9}\right)$, and for high neutral density $\left(10^{23} \mathrm{~m}^{-3}\right)$ at comparatively smaller values of ratio $N_{\mathrm{e}} / N_{\mathrm{i}}(0.99 \times$

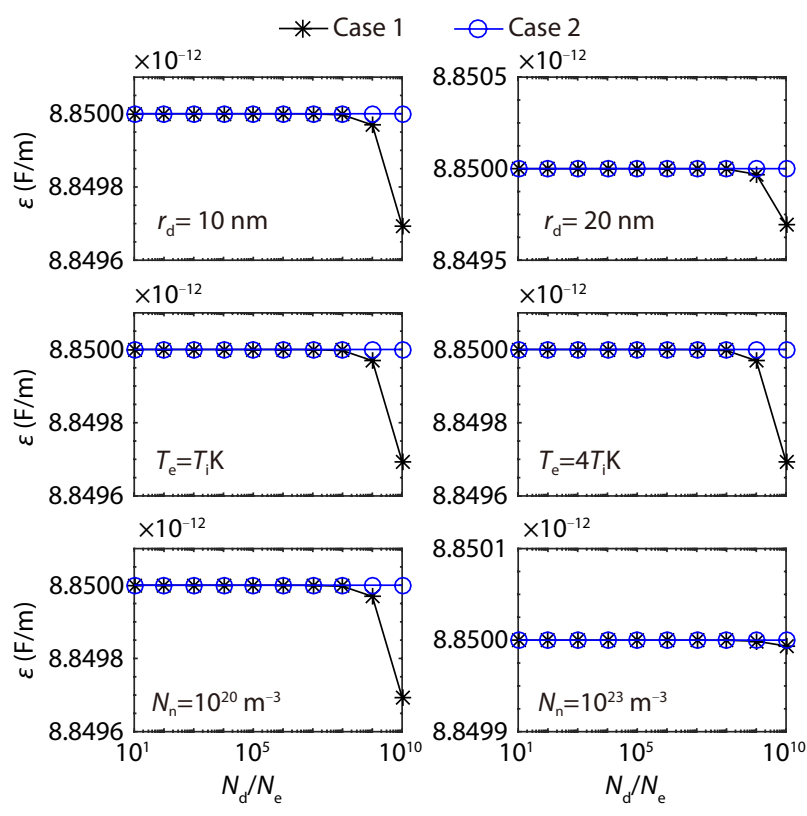

Figure 6. Dependence of complex permittivity on dust-to-electron density ratio $\left(N_{\mathrm{d}} / N_{\mathrm{e}}\right)$ for different parameters and cases.

$\left.10^{-10}\right)$, the contribution of ion effects for complex permittivity becomes significant.

\section{Discussion}

In this study we present modified equations of conductivity and permittivity with ion effects in a weakly-ionized dusty plasma, in 
low and high dust density. Relatively high dust density causes the electron bite-out condition because the dust particles act as an efficient sink for electrons (Rapp and Lübken, 2004). Figure 7 shows the reproduced experimental result recorded during the ECT-02 sounding rocket campaign (Havnes et al., 1996), with two distinct layers of electron bite-out centred at $87.5 \mathrm{~km}$ and $85.5 \mathrm{~km}$ altitudes with two corresponding distinct layers of charged dust particles exactly at the same altitudes (Havnes et al., 1996).
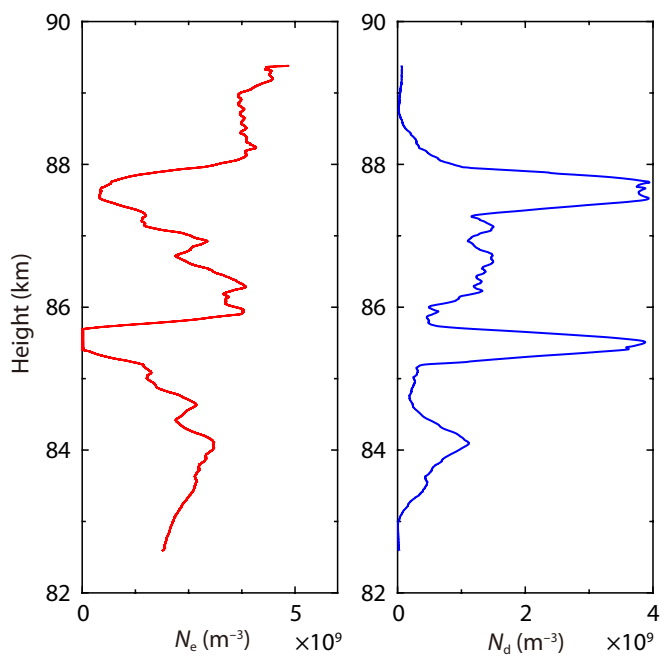

Figure 7. Height profiles of electron number density and charged dust number density measured during sounding rocket flight ECT-02 (Havnes et al., 1996).

Considering only the ion parameters from complex conductivity and permittivity Equations (25) and (26), respectively:

$$
\left.\begin{array}{l}
v_{\mathrm{Ti}}=\sqrt{K T_{\mathrm{i}} / m_{\mathrm{i}}} \\
v_{\text {eff_ion }}=N_{\mathrm{n}} \pi r_{\mathrm{d}}{ }^{2} v_{\mathrm{Ti}} \\
\omega_{\mathrm{pi}}=\sqrt{e^{2} N_{\mathrm{i}} / \varepsilon_{0} m_{\mathrm{i}}} \\
\eta_{\mathrm{id}}=\frac{e^{2} \pi r_{\mathrm{d}}{ }^{2} N_{\mathrm{i}} N_{\mathrm{d}}}{m_{\mathrm{i}}}
\end{array}\right\},
$$

due to very small effects, the charging frequency of ion $v_{\mathrm{ch}}$ is not considered in this set of parameters. These parameters are smaller than their corresponding electron parameters because of the high mass of ions. In the absence of the electron bite-out condition, where the dust density $\left(N_{\mathrm{d}}\right)$ is low and $N_{\mathrm{e}} \approx N_{\mathrm{i}}$, the changes in all the parameters in set of Equations (27) are insignificant. Therefore, in this case, the ion conductivity $\left(\sigma_{\mathrm{i}}\right)$ and permittivity $\left(\varepsilon_{\mathrm{i}}\right)$ effect can be ignored. However, in the presence of the electron biteout condition, where the dust density $\left(N_{\mathrm{d}}\right)$ is high and $N_{\mathrm{e}} \ll N_{\mathrm{i}}$, the plasma-ion frequency $\left(\omega_{\mathrm{pi}}\right)$ and charging response factor of ions $\left(\eta_{\text {id }}\right)$ increases. Therefore, in the bite-out condition, the presence of $\sigma_{\mathrm{i}}$ and $\varepsilon_{\mathrm{i}}$ significantly affects the results.

Our results indicate that even in the presence of the electron biteout condition, the ion effect on complex conductivity and permittivity is significant. However, for different dusty plasma parameters, the response of the bite-out condition is not the same (see Figures 1-2 and 4-5). In Figure 1 there is no bite-out whereas in
Figure 2 there is bite-out yet we still see that the results are almost the same. Similarly, in Figure 4 there is no bite-out, whereas in Figure 5 there is bite-out, and still we see that the results in Figure 4-5 are almost the same. This is because the bite-out conditions shown in Table 1 and used in Figures 2 and 5 are limited. Therefore, the increase in charging response factor and plasmaion frequency is comparatively small, and consequently, the ion effect on complex conductivity and permittivity also remains comparatively small. On the other hand in Figures 3 and 6, the results of cases 1 and 2 show variation that grows more obvious as long as the ratio $N_{\mathrm{d}} / N_{\mathrm{e}}$ increases. In this case, due to comparatively high dust density, the increase in charging response factor and plasma-ion frequency is comparatively high, and consequently the ion effect on complex conductivity and permittivity is also very clear. This means in the absence of the bite-out condition, the ions have no effect on conductivity and permittivity because the increases in charging response factor of ions, plasmaion frequency and effective collision frequency of ions are very small. In limited bite-out conditions, the contribution of ions in the equations of conductivity and permittivity may have even less significance. In contrast, with increasing dust density (ion density) and decreasing electron density, the bite-out becomes stronger, and the ion effect on conductivity and permittivity becomes clearly significant due to the increases in charging response factor of ions, plasma-ion frequency, and effective collision frequency of ions, which are high enough to contribute in the equations of conductivity and permittivity of dusty plasmas.

\section{Conclusions}

In this study, the effect of ions on complex conductivity and permittivity for dusty plasmas in the PMSE region (80-90 km) were studied, and the equations of complex conductivity and permittivity were modified as a result. Due to mesospheric changes in the PMSE altitude region, an electron bite-out is formed which contains high dust density as compared to electron density. From simulation results, we found that the ion effect on conductivity becomes significant in the comparatively strong bite-out condition for smaller dust size, smaller electron temperature, and smaller neutral density. The ion effect on conductivity becomes significant in the comparatively weak bite-out condition for larger dust size, higher electron temperature, and higher neutral density. Moreover, for different dust sizes, electron temperatures and neutral densities, the ion effects on complex permittivity become significant only in the very strong bite-out condition.

Based on the simulation results, it is concluded that without the electron bite-out condition, the effect of ions on conductivity and permittivity is not significant and can be neglected. However, in the bite-out condition, the effect of ions on conductivity and permittivity shows significant effects which cannot be ignored.

\section{Acknowledgments}

This work is supported by the National Natural Science Foundation of China (No. 61671116, 61771096, 11905026), National Key Research and Development Program of China (No. 2019YFA02 10202) and Fundamental Research Funds for the Central Universities (No. ZYGX2019Z006, ZYGX2019J012). We also thank Pro- 
fessor M. RAPP and O. HAVNES for providing the data of ECT-02. The EISCAT Scientific Association is supported by the research councils of China, Finland, France, Germany, Japan, Norway, Sweden and UK.

\section{References}

Biebricher, A., and Havnes, O. (2012). Non-equilibrium modeling of the PMSE Overshoot Effect revisited: a comprehensive study. J. Plasma Phys., 78(3), 303-319. https://doi.org/10.1017/S0022377812000141

Biebricher, A., Havnes, O., and Bast, R. (2012). On the necessary complexity of modeling of the polar mesosphere summer echo overshoot effect. J. Plasma. Phys., 78(3), 225-239. https://doi.org/10.1017/S0022377811000596

Blix, T. A. (1999). The importance of charged aerosols in the polar mesosphere in connection with noctilucent clouds and polar mesosphere summer echoes. Adv. Space Res., 24(12), 1645-1654. https://doi.org/10.1016/S02731177(99)00332-4

Chen, C., and Scales, W. A. (2005). Electron temperature enhancement effects on plasma irregularities associated with charged dust in the Earth's mesosphere. J. Geophys. Res., 110(A12), A12313. https://doi.org/10.1029/2005JA011341

Chilson, P. B., Belova, E., Rietveld, M. T., Kirkwood, S., and Hoppe, U. P. (2000). First artificially induced modulation of PMSE using the EISCAT heating facility. Geophys. Res. Lett., 27(23), 3801-3804. https://doi.org/10.1029/2000GL011897

Duan, J. Z., Wang, C. L., Zhang, J. R., Ma, S. Q., Hong, X. R., Sun, J. A., Duan, W. S., and Yang, L. (2012). Influence of charging process and size distribution of dust grain on the electric conductivity of dusty plasma. Phys. Plasmas, 19(8), 083703. https://doi.org/10.1063/1.4744972

Friedrich, M., Torkar, K. M., Singer, W., Strelnikova, I., Rapp, M., and Robertson, S. (2009). Signatures of mesospheric particles in ionospheric data. Ann. Geophys., 27(2), 823-829. https://doi.org/10.5194/angeo-27-823-2009

Ge, S. C., Li, H. L., Meng, L., Wang, M. Y., Xu, T., Ullah, S., Rauf, A., and Abdel, H. (2020). On the radar frequency dependence of polar mesosphere summer echoes. Earth Planet. Phys., 4(6), 571-578.

Ginzburg, V. L. (1970). The Propagation of Electromagnetic Waves in Plasmas. Oxford: Pergamon.

Havnes, O., Melandsø, F., La Hoz, C., Aslaksen, T. K., and Hartquist, T. (1992). Charged dust in the Earth's mesopause; effects on radar backscatter. Phys. Scr., 45(5), 535-544. https://doi.org/10.1088/0031-8949/45/5/022

Havnes, O., Trøim, J., Blix, T., Mortensen, W., Næsheim, L. I., Thrane, E., and Tønnesen, T. (1996). First detection of charged dust particles in the Earth's mesosphere. J. Geophys. Res., 101(A5), 10839-10847. https://doi.org/10.1029/96JA00003

Havnes, O., La Hoz, C., Næsheim, L. I., and Rietveld, M. T. (2003). First observations of the PMSE overshoot effect and its use for investigating the conditions in the summer mesosphere. Geophys. Res. Lett., 30(23), 2229. https://doi.org/10.1029/2003GL018429

Havnes, O. (2004). Polar mesospheric summer echoes (PMSE) overshoot effect due to cycling of artificial electron heating. J. Geophys. Res., 109(A2), A02309. https://doi.org/10.1029/2003JA010159

Jana, M. R., Sen, A., and Kaw, P. K. (1993). Collective effects due to chargefluctuation dynamics in a dusty plasma. Phys. Rev. E, 48(5), 3930-3933. https://doi.org/10.1103/PhysRevE.48.3930

Jia, J. S., Yuan, C. X., Gao, R. L., Wang, Y., Liu, Y. Z., Gao, J. Y., Zhou, Z. X., Sun, X. D., Wu, J., ... and Pu, S. Z. (2015). Propagation of electromagnetic waves in a weakly ionized dusty plasma. J. Phys. D: Appl. Phys., 48(46), 465201. https://doi.org/10.1088/0022-3727/48/46/465201

Kassa, M., Havnes, O., and Belova, E. (2005). The effect of electron bite-outs on artificial electron heating and the PMSE overshoot. Ann. Geophys., 23(12), 3633-3643. https://doi.org/10.5194/angeo-23-3633-2005
Lübken, F. J. (1999). Thermal structure of the Arctic summer mesosphere. J. Geophys. Res., 104(D8), 9135-9149. https://doi.org/10.1029/1999JD900076

Ma, J. X., and Yu, M. Y. (1994). Langmuir wave instability in a dusty plasma. Phys. Rev. E, 50(4), R2431-R2434. https://doi.org/10.1103/PhysRevE.50.R2431

Mahmoudian, A., and Scales, W. A. (2012). Temporal evolution of radar echoes associated with mesospheric dust clouds after turn-on of radio wave heating. J. Geophys. Res., 117(D6), D06221. https://doi.org/10.1029/2011JD017166

Næsheim, L. I., Havnes, O., and La Hoz, C. (2008). A comparison of polar mesosphere summer echo at VHF $(224 \mathrm{MHz})$ and UHF $(930 \mathrm{MHz})$ and the effects of artificial electron heating. J. Geophys. Res., 113(D8), D08205. https://doi.org/10.1029/2007JD009245

Rapp, M., and Lübken, F. J. (2000). Electron temperature control of PMSE. Geophys. Res. Lett., 27(20), 3285-3288. https://doi.org/10.1029/2000GL011922

Rapp, M., and Lübken, F. J. (2004). Polar mesosphere summer echoes (PMSE): review of observations and current understanding. Atmos. Chem. Phys., 4(11-12), 2601-2633. https://doi.org/10.5194/acp-4-2601-2004

Rauf, A., Li, H. L., Ullah, S., Meng, L., Wang, B., and Wang, M. Y. (2018). Investigation of PMSE dependence on high energy particle precipitation during their simultaneous occurrence. Adv. Space Res., 63(1), 309-316. https://doi.org/10.1016/j.asr.2018.09.007

Routledge, G., Kosch, M. J., Senior, A., Kavanagh, A. J., McCrea, I. W., and Rietveld, M. T. (2011). A statistical survey of electron temperature enhancements in heater modulated polar mesospheric summer echoes at EISCAT. J. Atmos. Solar-Terr. Phys., 73(4), 472-482. https://doi.org/10.1016/j.jastp.2010.11.004

Scales, W. (2004). Electron temperature effects on small-scale plasma irregularities associated with charged dust in the Earth's mesosphere. IEEE Trans. Plasma Sci., 32(2), 724-730. https://doi.org/10.1109/tps.2004.826082

Scales, W. A., and Mahmoudian, A. (2016). Charged dust phenomena in the near-Earth space environment. Rep. Prog. Phys., 79(10), 106802. https://doi.org/10.1088/0034-4885/79/10/106802

Senior, A., Mahmoudian, A., Pinedo, H., La Hoz, C., Rietveld, M. T., Scales, W. A., and Kosch, M. J. (2014). First modulation of high-frequency polar mesospheric summer echoes by radio heating of the ionosphere. Geophys. Res. Lett., 41(15), 5347-5353. https://doi.org/10.1002/2014GL060703

Shi, Y. X., Ge, D. B., and Wu, J. (2007). Theoretical analysis of microwave attenuation constant of weakly ionized dusty plasma. Chin. J. Geophys., 50(4), 877-883. https://doi.org/10.1002/cjg2.1105

Shukla, P. K., and Mamun, A. A. (2002). Introduction to Dusty Plasma Physics. Bristol: Institute of Physics.

Singer, W., Latteck, R., Friedrich, M., Wakabayashi, M., and Rapp, M. (2011). Seasonal and solar activity variability of D-region electron density at $69^{\circ} \mathrm{N}$. J. Atmos. Solar-Terr. Phys., 73(9), 925-933. https://doi.org/10.1016/j.jastp.2010.09.012

Ulwick, J. C., Baker, K. D., Kelley, M. C., Balsley, B. B., and Ecklund, W. L. (1988). Comparison of simultaneous MST radar and electron density probe measurements during STATE. J. Geophys. Res., 93(D6), 6989-7000. https://doi.org/10.1029/JD093iD06p06989

Von Zahn, U., and Meyer, W. (1989). Mesopause temperatures in polar summer. J. Geophys. Res., 94(D12), 14647-14651. https://doi.org/10.1029/JD094iD12p14647

Von Zahn, U., and Bremer, J. (1999). Simultaneous and common-volume observations of noctilucent clouds and polar mesosphere summer echoes. Geophys. Res. Lett., 26(11), 1521-1524. https://doi.org/10.1029/1999gl900206

Weingartner, J. C., and Draine, B. T. (2001). Photoelectric emission from interstellar dust: grain charging and gas heating. Astrophys. J. Suppl. Ser., 134(2), 263-281. https://doi.org/10.1086/320852 